International Business Management 4 (2): 89-98, 2010

ISSN: $1993-5250$

(C) Medwell Journals, 2010

\title{
Impact of Accreditation on Human Resource Development and Management of a Hospital Through HR Balance Score Card
}

\author{
${ }^{1}$ Syed Jaleeluddin Hyder, ${ }^{2}$ Bimal Kumar Mishra and ${ }^{3}$ Manju Bhagat \\ ${ }^{1}$ Health Solution Management Consultancy, P.O. Box 215, Rehab, Kuwait \\ ${ }^{2}$ Department of Applied Mathematics, Birla Institute of Technology, Mesra 835215, Ranchi, India \\ ${ }^{3}$ Department of Management, Birla Institute of Technology, Mesra 835215, Ranchi, India
}

\begin{abstract}
The number of institutions using Accreditation as a tool to improve the quality of their processes and professionals has grown over the years but their real impact is questionable. The present study uses the HR Balance Scorecard to monitor and improve the quality of HR processes for the development and management of its professionals in a hospital. The processes of HR balance scorecard included all facets of HR namely Compensation and Benefits, Recruitment and Manpower Planning, Retention and Turnover, Occupational Health and Safety and HR Department Efficiency and Achievements. There has been remarkable improvement in all the listed processes which was confirmed by the satisfaction surveys conducted with the employees as well as managers. HR Balance Scorecard objectively guided the level of improvement and also highlighted the grey areas of improvement.
\end{abstract}

Key words: HR balance score card, impact of accreditation, human resource development, human resource management

\section{INTRODUCTION}

Accreditation Canada International promotes accreditation around the world. Recognizing that one standard approach does not work for everyone, Accreditation Canada International customizes its accreditation program to meet the client's needs (Canada, 2006). Working collaboratively with clients, Accreditation Canada provides continuous guidance without being prescriptive. Accreditation is not a pass or fail.

Accreditation means helping organizations prepare for success. Accreditation Canada's accreditation program is adaptable, culturally sensitive and competitively priced. Implementation of the accreditation program and cycle is flexible depending on the client's level of readiness. The human resources development and management standards provide guidance to clients to assess and benchmark their processes against the standards.

Despite the limited but growing evidence base on the impact of HRM on organizational performance in other sectors, there have been relatively few attempts to assess the implications of this evidence for the health sector. Buchan (2004) examines this broader evidence base on HRM in other sectors and examines some of the underlying issues related to good HRM in the health sector. Traditionally, the Balanced Scorecard (BSC) has been an effective tool in linking measurement to strategy. However, what is least understood is how the BSC can be used to redefine organizational relationships, re-engineer fundamental processes and transform organizational culture for superior performance in an organization with the same people, services and technology that previously delivered dismal performance. Tsasis and Harber (2008) highlight the process and uses York Central Hospital in Toronto, Ontario, Canada as an illustrative example.

Human resource competency becomes key factor in public hospitals that needs an overall improvement. For achieving this aim, the study by Sitawati et al. (2009) describes human resources performance measurement steps that comprises of preparing Activity Value Chain, Job Description, Job Specification, Job Performance Standard and Job Performance Scorecard.

Those steps had been implemented in public hospitals in NTT province, Indonesia and its expected that the measures taken would enhance the organizations performance. Schuler and Jackson (2006) covers issues of globalization and knowledge management and their effect on the field of Human Resource Management (HRM) and Strategic Human Resource Management (SHRM) to achieve global competitive edge. They also explore the new reality of knowledge management and its

Corresponding Author: Syed Jaleeluddin Hyder, Health Solution Management Consultancy, P.O. Box 215, Rehab, Kuwait 
implications for HRM and SHRM. Gifford et al. (2002) conducted a study that investigates the relationships between unit organizational culture and several important job related variables for nurse retention in the labor and delivery units of seven hospitals. Data analysis shows that unit organizational culture does affect nurses quality of work life factors and that human relation cultural values are positively related to organizational commitment, job involvement, empowerment and job satisfaction and negatively related to turnover.

These findings suggest that although increasing recruitment of nurses and improved compensation and benefits strategies may offset hospital nurses shortages in the short term improving quality of work life may be a more practical and long term approach to improving hospital nurse retention.

The competency framework has attracted interest in the business world at a time when organisations are faced with the pressing need to develop the right calibre of people and particularly their managers. Organisations have always been striving to develop the effective manager. Nowadays the demand to achieve this goal rests on the rate of change which necessitates continuous transformation. Antonacopoulou and Gerald (1996) present's a competency framework as a basis of management development activities in an attempt to develop productive and effective manager.

Holbeche (2001) shows how HR strategists have impressed and delivered at the highest level in shaping the growth and direction of the organizations and hence achieving a variety of key business objectives. The study focuses on performance and developmental issues which are key to aligning HR and Business strategies and shows how HR strategists have leveraged their role to deliver key business objectives. Case studies include managing change, influencing organizational development, identifying and retaining talent and leadership development.

\section{MATERIALS AND METHODS}

HR balance score card: The requirement for an effective and efficient Human Resource Management is stronger than ever. As a result, the HR best practices have become the need of the hour for improving satisfaction, increasing productivity and assuring retention through the provision of quality programs, policies and initiatives.

The objective is to create a work force of 20 th Century by observing higher level of efficiency, timeliness and appropriateness so as to improve the outcomes of Human Resource Development and Management through
HR Balanced Scorecard. Although, the application of a complete balanced scorecard is one of the most common tool in Strategic Management to gauge the performance of business but there is still lack of focus on its application in Human Resources Management and Development. This is particularly critical for the survival of business as human resource now has become the main organization asset in this competitive world.

The Balanced Scorecard forms a conceptual measurement model for assessing the Human Resources Development and Management where this model complements numerical measures of past performance with measures of current performance. Some of the attributes of measurements include Responsive processes, Innovation, Creativity and Productivity.

By having Balanced Standard will decrease evaluator subjectivity so that the evaluated processes or outcomes have the same guidance in understanding the performance. All the processes or outcomes are benchmarked to certain numerical or qualitative perspective and then the actual processes or outcomes are measured against the set criteria and then judged as either Lagging or Leading Indicator through different color codes. The processes were further classified as either High Risk, High Volume or Problem Prone depending on their nature and their impact. The HR Scorecard would assist the HR Department as well the Management in the following manner:

- The extent of HR efforts, initiatives and their success rate

- Measure the results or outcomes of HR, Training, Performance Assessment etc.

- Tracking vital indicators gives early warning signals of organizational concerns, ignoring these concerns may lead to organizational collapse

The HR Scorecard enables the employees to:

- Stay connected with the management in order to address their concerns

- The employees would gain timely feedback to their desires and concerns

- Gives a sense of leadership and organizational accountability

- Promotes employees towards organizational alignment and sense of attachment to its mission and vision

\section{RESULTS AND DISCUSSION}

Employees being the heart of every business process and every transaction in health care environment, the management in collaboration with $\mathrm{HR}$ department have 
taken steps for not only retaining key talent but also rewarding employees through measurable objectives. A comprehensive talent management strategy is developed by managing and monitoring indicators as part of the Accreditation process through HR Balanced Scorecard.

\section{Compensation and Benefits \\ Compensation:}

- Average increase in total compensation of Physicians by $81 \%$, Nursing 50\%, Technicians $29 \%$ and Support Service Staff by $25 \%$

\section{Benefits:}

- Average increase in benefits of assistant registrars by $47 \%$, Registrar $38 \%$, Specialist $28 \%$ and Consultants by $31 \%$

- Average increase in benefits of Nursing supervisor by $25 \%$, Head Nurse $24 \%$ and Staff Nurse $21 \%$

- Average increase in benefits of managers by $26 \%$, Supervisor $30 \%$ and Team Member 29\%

\section{Benchmarking index of compensation:}

- Benchmarking index of assistant registrars by 1.09 , Registrar 1.25, Specialist 1.15 and Consultants by 1.20

- Benchmarking index of nurse manager 1.07, Head Nurse 1.11, Staff Nurse 1.07 and Care Giver 1.09

- Benchmarking index of lab Technician 1.08, Laboratory Assistant Technician 1.15, Radiology Technician 0.95 and MRI Technician 0.90

- Benchmarking index of Manager 1.21, Supervisor 1.20 and Secretary 1.00

\section{Absenteeism cost and rate:}

- Absenteeism rate was reduced for nursing from $2.21-1.52 \%$ and for Support Service Staff from $1.37-1.01 \%$

- On the other hand there was rise in Absenteeism Cost for Nursing by $27 \%$ and for Support Service Staff by $16 \%$

\section{Promotions and increments:}

- $44 \%$ of doctors were promoted or given increment

- $89 \%$ of nursing were promoted or given increment

- $68 \%$ of technicians were promoted or given increment

- $63 \%$ of support service staff were promoted or given increment

Conditions of service were quite outdated at the start of 2007, the accreditation process and the standards paved the way for Clients needs assessment and were further elucidated through the Staff Satisfaction Surveys.
At the start of the process Compensation and Benefits was classified as high risk and problem prone process as it affects the retention of employees and in turn the stability of the organization.

Hence the Conditions of service were revised and Benchmarked against Local and International Industry Standards. Its observed that the Organization has become one of the best payers in terms of Compensation as well as Benefits. More than $60 \%$ of the employees have said they would prefer the present organization when compared to its competitors for their own stability and growth. The Organization and the HR Department has performed exceeding well on this front and achieved or exceeded nearly all the HR Scorecard benchmark levels set as part of Compensation and Benefits.

\section{Recruitment and manpower planning Recruitment cost:}

- $93 \%$ reduction in Recruitment Cost from year 2007 as against 2009 and Average Cost per Recruitment KD 203

- $9 \%$ reduction in Recruitment Cost from year 2008 as against 2009 and Average Cost per Recruitment KD 254

- Average Cost per Recruitment in 2009 is KD 26

\section{Good hires:}

- $87 \%$ of the HOD's including Medical Director were satisfied with the hiring of Physicians

- $73 \%$ of the Nursing Supervisors including Nursing Director were satisfied with the hiring of Nurses

- 76\% the HOD's including Lab and Radiology Directors were satisfied with the hiring of Technicians

- $68 \%$ of the Managers were satisfied with the hiring of Support Service Staff

There is no record of satisfaction rates of old hires although many HOD's and Supervisors were concerned with the competency levels of the new hired staff (i.e., for years 2007 and 2008). A survey was conducted to estimate the success of hiring through web and all the HOD's and Directors have expressed their satisfaction with the new hires as discussed earlier.

\section{Recruitment lag:}

- $50 \%$ reduction in Recruitment Lag from year 2007 as against 2009 for Physicians

- $67 \%$ reduction in Recruitment Lag from year 2007 as against 2009 for Nursing

- $67 \%$ reduction in Recruitment Lag from year 2007 as against 2009 for Technicians

- $75 \%$ reduction in Recruitment Lag from year 2007 as against 2009 for Support Service Staff 
- $46 \%$ reduction in Recruitment Lag from year 2007 as against 2009 for Managers

- $67 \%$ reduction in Recruitment Lag from year 2007 as against 2009 for Supervisors

- $72 \%$ reduction in Recruitment Lag from year 2007 as against 2009 for Team Members

Normal vs. web resume processing duration:

- $50 \%$ reduction in Resume Processing time from Normal Vs Web for Physicians

- $57 \%$ reduction in Resume Processing time from Normal $\mathrm{Vs}$ Web for Nursing

- $57 \%$ reduction in Resume Processing time from Normal $\mathrm{Vs}$ Web for Technicians

- $60 \%$ reduction in Resume Processing time from Normal Vs Web for Support Service Staff

- $29 \%$ reduction in Resume Processing time from Normal $\mathrm{Vs}$ Web for Managers

- $47 \%$ reduction in Resume Processing time from Normal $\mathrm{Vs}$ Web for Supervisors

- $71 \%$ reduction in Resume Processing time from Normal Vs Web for Team Members

Benchmarking ratio of manpower to an hospital of similar size and nature of specializations:

- Benchmark ratio for Physicians is 0.69. There are plans for recruiting more doctors as its affecting the safety and patient waiting time (in turn patient satisfaction)

- Benchmark ratio for Nursing is 1.38

- Benchmark ratio for Technicians is 0.88

- Benchmark ratio for Support Services Staff is 1.06

HR budget compliance: HR Budgeting started from 2009 and the compliance has been at satisfactory rates. Initially there being no HR Budget there was no control on the hiring as well as additional expenses like overtime, or other allowances. Moreover, the HR Budget is strictly being monitored by the Management owing to Financial Crisis. The HR Budget includes basic salary, bonuses, benefits and new hire salaries. The HR Budget compliance rates are listed for different categories:

- Physicians: $77 \%$

- Nursing: 95\%

- Technicians: 83\%

- Support Services: $79 \%$

There has been remarkable improvement in terms of Recruitment where both Recruitment Cost and Lag has been reduced dramatically. Initially it was a major dissatisfaction among the Department Heads as the vacancy fulfillment time was huge but the efforts HR took is paying off and its been able to provide the right candidate at the right time as stipulated in the Manpower plan or as required. Again HR has performed far above expectations and broken all HR Scorecard benchmark levels set as part of Recruitment and Manpower Planning.

\section{Retention and turnover}

Turnover rates:

- Turnover rates have reduced from $17 \%$ in year 2007 to 9\% in Year 2009 for Physicians

- Turnover rates have reduced from $9 \%$ in year 2007 to $3 \%$ in year 2009 for Nursing

- Turnover rates have increased from $17 \%$ in year 2007 to $21 \%$ in year 2009 for Technicians

- Turnover rates have reduced from 24\% in year 2007 to 3\% in Year 2009 for Support Service Staff

- Overall Turnover rates have reduced from $17 \%$ in year 2007 to $9 \%$ in year 2009

The reason for highest turnover rates of technicians especially the Radiology and MRI could be correlated to their salaries offered by the competitors (The compensation bench mark index stood at 0.90). Moreover, its very difficult to get a licensed Radiology and MRI technician in this part of the world; hence the competitors are on the look out to grab them with higher salaries and attractive benefits.

Employee satisfaction rates: Employee satisfaction has been the highest priority of the management and strives to provide the best to its staff. The flexible timings enable staff to work out a balance between personal and professional obligations. The succession work plan provides staff with necessary impetus to perform and there is always a reward for good work.

HR believes in good work pays and good performers are recognized for their contribution during the Organizations Anniversary celebrations. Apart from the professional aspect, the personal needs of staff are also met in the form of furnished apartments, family related leave arrangements etc. There has been significant increase in staff satisfaction over the years as shown in Table 1 . The yearly staff satisfaction survey provides the organization the direction to upgrade its staff assistance programs.

\begin{tabular}{lccc}
\multicolumn{4}{l}{ Table 1: Employee satisfaction rates } \\
Profession & $\begin{array}{c}\text { Satisfied } \\
(2007, \mathrm{n}=327)\end{array}$ & $\begin{array}{c}\text { Satisfied } \\
(2008, \mathrm{n}=321)\end{array}$ & $\begin{array}{c}\text { Satisfied } \\
(2009, \mathrm{n}=378)\end{array}$ \\
\hline Doctors & $30 \%$ & $41 \%$ & $45 \%$ \\
Nurses & $68 \%$ & $75 \%$ & $74 \%$ \\
Technicians & $40 \%$ & $59 \%$ & $66 \%$ \\
Support services & $42 \%$ & $48 \%$ & $70 \%$ \\
Overall satisfaction & $53 \%$ & $64 \%$ & $68 \%$ \\
\hline
\end{tabular}




\section{Cost of turnover:}

- $13 \%$ reduction in Cost of Turnover from year 2007 as against 2009 for Physicians

- $42 \%$ reduction in Cost of Turnover from year 2007 as against 2009 for Nursing

- $\quad 90 \%$ increase in Cost of Turnover Lag from year 2007 as against 2009 for Technicians

- $79 \%$ reduction in Cost of Turnover Lag from year 2007 as against 2009 for Support Service Staff

Efforts taken by the Management and HR have paid dividends and there is reduction in Cost of turnover except for Technician Staff. Once the Technicians salary scale would be revised there would be substantial improvement in the Cost of Turn Over for Technicians.

Succession planning: The objective is to develop a workforce planning system (Table 2) that meets the specific needs of the organization and its respective units and is fully aligned with Organizations strategic, business and administrative (budget, recruiting and employee development) systems. Few other objectives of Succession Planning are:

- Focus is on key positions/areas at various levels

- Focus is on the development of talent for the longer term

- Plans include developing pools of talent for key positions/areas

- Plans are linked to building competencies and skills for current and future business needs

\begin{tabular}{|c|c|c|c|c|c|}
\hline $\begin{array}{l}\text { Total } \\
\text { managers }\end{array}$ & $\begin{array}{l}\text { Total succession } \\
\text { plans created }\end{array}$ & Rate & $\begin{array}{c}\text { Other senior/ } \\
\text { critical positions } \\
\text { identified }\end{array}$ & $\begin{array}{c}\text { Succession } \\
\text { plans } \\
\text { created }\end{array}$ & Rate \\
\hline 28 & 18 & $64 \%$ & 10 & 3 & $30 \%$ \\
\hline
\end{tabular}

- A systematic process is used to assess candidates based on feedback from multiple perspectives and sources of information

- Processes are being developed to integrate succession planning with other HR activities

Further to illustrate the progress of Succession Planning Culture a survey was conducted to assess the strategies aiding from Managers and Management perspective that are shown in Fig. 1 and 2.

Occupational health and safety: Job/Equipment hazards were created by respective divisions as shown in Table 3 and all relevant employees were provided orientation of the same as a result the number of incidents or adverse events has reduced considerably. Impact of Orientation and Training and Job Hazard Analysis on Injuries:

- $50 \%$ reduction in Falls/Slips Injuries from year 2008 as against 2009

- $56 \%$ reduction in Sharp or Needle pricks Injuries from year 2008 as against 2009

- $50 \%$ reduction in Injuries from Equipments from year 2008 as against 2009

- $100 \%$ reduction in Life Threatening Injuries from year 2008 as against 2009

- $100 \%$ reduction in Injuries from Falling Objects from year 2008 as against 2009

\section{Further health and wellness initiatives:}

- 'I quit smoking program' was offered to employees free of cost and there were about 15 employees who registered for such an initiative and the success rate was phenomenal i.e., $93 \%$

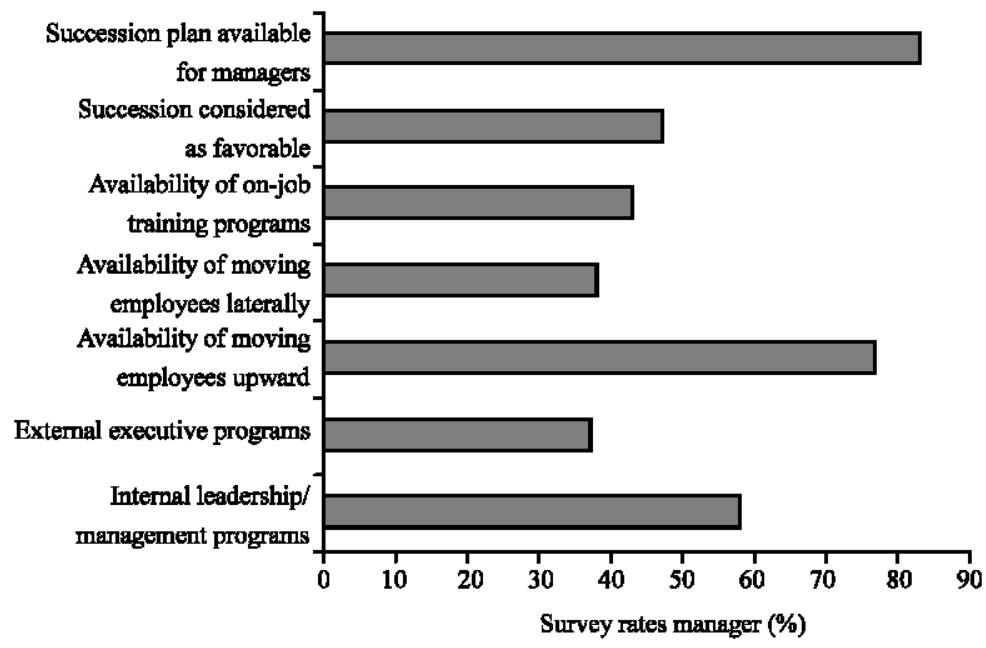

Fig. 1: Strategies aiding succession planning manager's survey response 
Int. Business Manage., 4 (2): 89-98, 2010

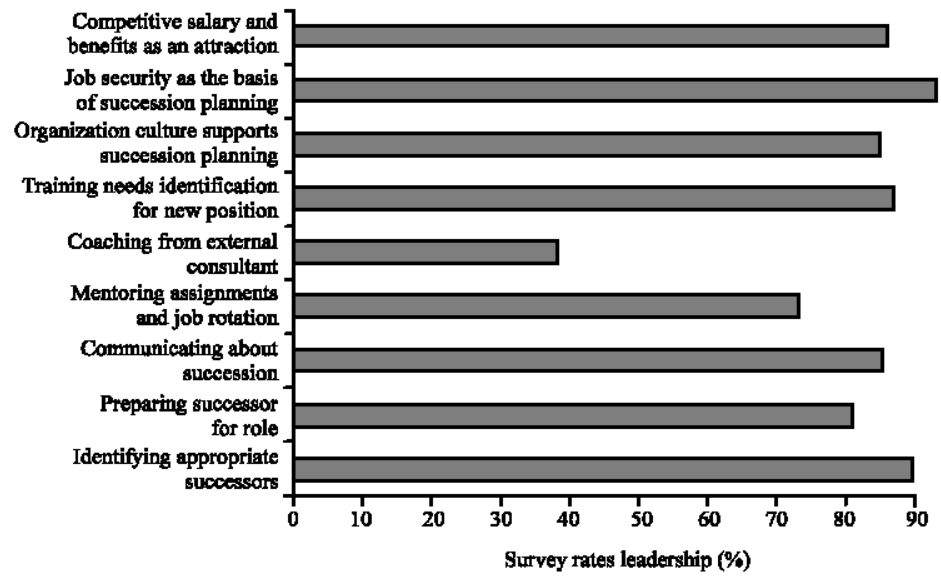

Fig. 2: Strategies aiding succession planning management survey response

Table 3: Job/equipment hazards created and orientation given to employee

\begin{tabular}{lr}
\hline Hazards & 2009 \\
\hline Bio-medical & 25 \\
Radiology & 9 \\
Laboratory & 4 \\
Environmental services & 13 \\
Maintenance & 3 \\
Total & 54 \\
\hline
\end{tabular}

- Hospital information system tickler system was used to remind the employees of Diabetes checkups and $83 \%$ responded there by aiding them in keeping their diabetes in control

- Gym: All employees have access to free Gym, most of the employees having Obesity, Diabetes of BP problems are regularly accessing Gym (As of now no record is available)

- Health Check-up: Complete health check-up is performed at the time of employment free of cost, though there is no mechanism for regular health checkups

- SPA: Access to SPA is offered at discounted rates where employees could relax and reduce their weights

Medical insurance: The Hospital provides Medical Services to its employees at a discounted rate. Consultation is free where as there is $50 \%$ discount for examinations and $10 \%$ discount on Pharmacy items. Applicable charges for the examinations and medicine are deducted from the employees salary. The doctor can provide sample medicines free of cost provided they are available. The Medical units also issues medical reports for sick leave and the residence permit and eye tests for the driving license.

Informal discussions with Staff and the Staff Satisfaction Survey have revealed Staff Medical Insurance to be a high priority need. This is in the process of organization through the Finance Department. Except
Medical Insurance there is good progress in all directions of Occupational Health and Safety and nearly meets all the benchmarks set in the HR Balance Scorecard (Fig. 3, Table 4).

\section{HR department efficiency and achievements Compensation and leave issues:}

- Payroll processing duration reduced from 3 days to 15 min with the implementation and customization of HRMS

- Payroll related errors reduced from about 20 to 1-3 month $^{-1}$ with the implementation of proper controls

- Compensation related errors/queries reduced by $77 \%$ with the implementation of pay slip

- Approval of Annual Leave reduced by 57\%, Short Leaves by $67 \%$, Conference Leaves by $50 \%$, Sick Leave by $67 \%$ either through Automation or removal of Signatories

Achievements of HR during 2007-2009: The HR plan encompasses the changing needs of its staff, the external and internal environment and need for leveraging technology. Various Initiatives were incorporated in the HR Plan namely Succession Planning, Training and Development, Performance Appraisal, Recruitment and Retention Strategies, Automation, Health and Safety Initiatives. The HR plan is customized to suit staff needs based on a needs assessment survey conducted annually. The employee handbook is an exceptional document that is developed to provide staff with a clear outline of the organizations mission, goals, objectives and strategic directions (Table 5). The document provides the vision, mission and beliefs of the organization and details employee rights and responsibilities. It also includes all pertinent information regarding leave, salaries, training 


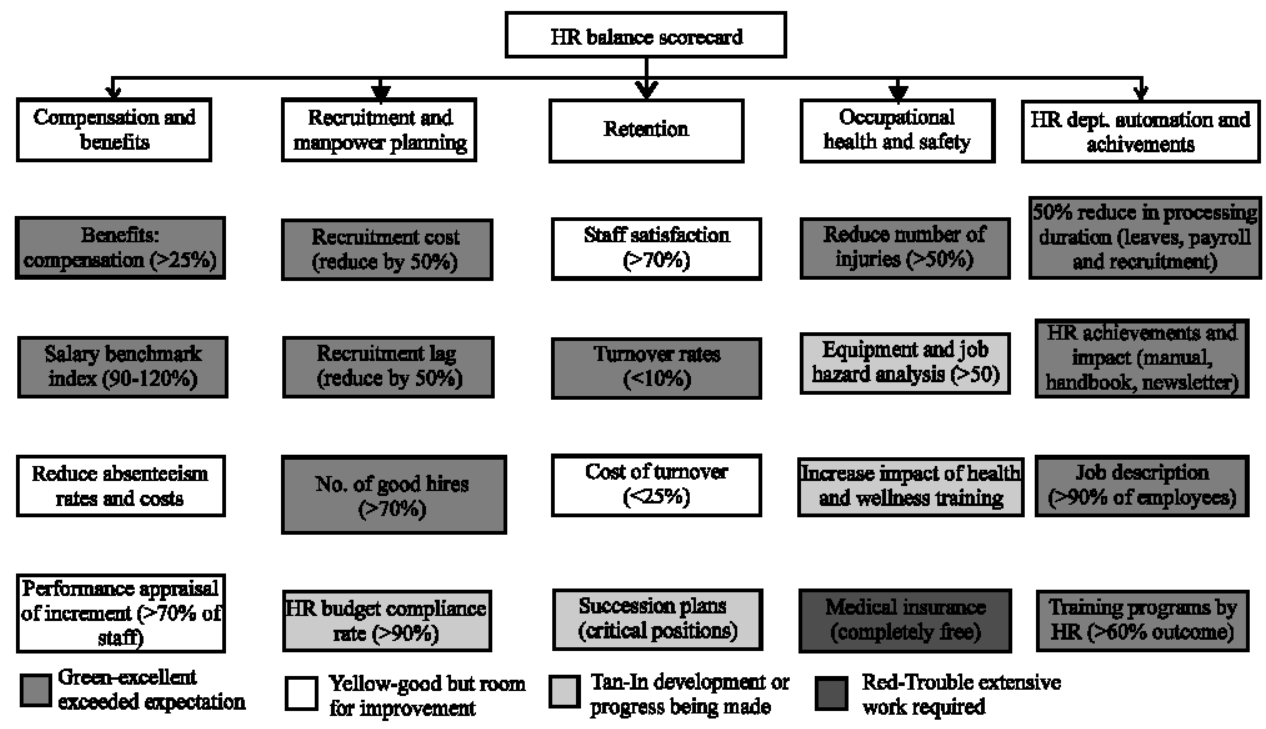

Fig. 3: The benchmarkes set in the HR balance scorecard

Table 4: Training sesssions in the realm of occupational health and safety

\begin{tabular}{|c|c|c|c|}
\hline Training modules & Sessions & Training modules & Sessions \\
\hline Fire and electrical safety & 9 & Accident investigation & 6 \\
\hline $\begin{array}{l}\text { Job and equipment } \\
\text { hazard analysis }\end{array}$ & 15 & $\begin{array}{l}\text { Protective equipment } \\
\text { and its advantages }\end{array}$ & 9 \\
\hline $\begin{array}{l}\text { Slip and fall } \\
\text { precautionary measures }\end{array}$ & 17 & $\begin{array}{l}\text { Physical, biological, } \\
\text { radiological hazards }\end{array}$ & 12 \\
\hline Ergonomics & 8 & Health and wellness & 18 \\
\hline Emergency preparedness & 11 & Health and safety audits & 3 \\
\hline Sharps handling & 17 & $\begin{array}{l}\text { Personal hygiene and } \\
\text { infection control }\end{array}$ & 21 \\
\hline $\begin{array}{l}\text { Incident reporting } \\
\text { Total }\end{array}$ & 17 & Disability management & $\begin{array}{r}6 \\
169\end{array}$ \\
\hline
\end{tabular}

and development, visa processing formalities, performance management, standards of behavior, employee grievance and disciplinary policies. The handbook is available in Arabic as well to ensure total staff coverage. Employee awareness on Policies and Procedures stood at $78 \%$ after the dissemination of an all inclusive comprehensive employee handbook.

The employee handbook initiative was appreciated by most of the employees. Several HR Policies, HR Forms, Physicians Duty Rosters were uploaded on the intranet for easy access by staff as a result of which almost $93 \%$ of staff access the Intranet to obtain the necessary HR forms. Medical By-Laws have been drafted approved and implemented that defines the framework and process for ensuring physician competence. Privileges are awarded to medical staff after verification of proof submitted, references from previous employer and on the job approval from experts, department head and credentialing and privileging committee. Privileges and renewal of privileges depend on position, licensure, prior experience and approval from the committee.
Table 5: HR achievments and their satisfaction levels among the employees \begin{tabular}{llll}
\hline Achievements & Satisfaction (\%) & Achievements & Satisfaction (\%)
\end{tabular}

\begin{tabular}{llll}
\hline HR plan & 78 & Medical insurance & 12
\end{tabular}

Employee handbook $\quad 95 \quad$ Occupational health $\quad 76$

$\begin{array}{lll}\text { Medical bylaws } & 73 & \text { and safety } \\ \text { Manpower plan } & 64\end{array}$

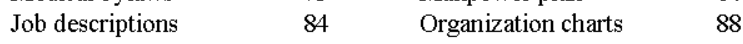

$\begin{array}{llll}\text { HR newsletter } & 88 & \text { HR manual } & 55\end{array}$

$\begin{array}{llll}\text { Automation } & 76 & \text { Duty rooster } & 98\end{array}$

Performance appraisal $\quad 83$

Job descriptions: About $82 \%$ of the job descriptions were created, approved and communicated to all the staff members. Employees were well aware of not only their present activities but their expectations for the coming year.

HR delves in depth the tools, materials and equipment used to perform the tasks in the job. For each job, the critical tasks and key result areas of the position are identified along with discrete outcomes of the job for which the person appointed will be held accountable and evaluated on.

As a result of which, HR in coordination with Department Heads have well developed job descriptions and specifications for almost $90 \%$ of staff who now clearly understands their roles, responsibilities, key deliverables and outcomes. Impact of Job Descriptions is shown in Table 6.

Its been observed that all categories of staff are extremely satisfied with the HR Newsletter, the first of its kind in the history of the organization. Nearly all sections of HR have been a success and the respondents highlighted some improvement areas that have been taken into consideration by HR Department as shown in 
Table 7. Feedback on quality improvement activities is also disseminated through HR Newsletter. Details are also uploaded on the intranet for maximum visibility and staff comments.

Orientation: About $88 \%$ of the new employees were satisfied with the Orientations offered to them in 2008 and $93 \%$ in 2009 . The orientation package is comprehensive and provides very good written material to new staff about the organization, its mission, programs, strategic plans, employee rights and responsibilities and mandatory training. Staff must complete a checklist of activities and demonstrate learning through written tests. The outcomes and the response from Orientees are shown in Table 8. Session on Standards of Behavior was imparted through role plays by employees which was informative in an innovative way.

Training: Training and development plans were established and implemented as employees rated them one among the top 3 needs in the Employee Satisfaction Survey. Management related and soft skills training were implemented for all clinical and support services staff. The training programs were interactive and encouraged staff participation through group discussions, brain storming, case analysis, presentations etc. HR Training plan is developed through staff needs

Table 6: Impact of job descriptions opinion of managers

\begin{tabular}{lc}
\hline Advantages of job descriptions & Percentage \\
\hline Job expectations & 88 \\
Defines scope of service & 95 \\
Better job performance & 78 \\
Defines skill set required & 95 \\
Aids in training needs assessment & 89 \\
Better customer service & 73 \\
Time and deadline management & 78 \\
Better accountability & 83 \\
Aids objectively in performance appraisal & 99 \\
Aids in quality management or initiatives & 93 \\
Enhances employee satisfaction & 88 \\
\hline
\end{tabular}

assessment Core competencies and specialized competencies were developed for each position category in collaboration with the respective Head of the Department and incorporated as part of Customized Training Sessions as shown in Table 9. The core skills are a mix of management, leadership, team building, change management and quality improvement initiatives.

This methodology has helped HR to offer Training programs of very high standards and also provided the employee impetus to perform better and exceed Managers expectations. Staffs are encouraged to evaluate training effectiveness and concepts presentation by trainer. Employee suggestions and feedback are used during the planning phase of future training activities. On the job impact of Trainings offered is shown in Table 10.

Grievance resolution: $H R$ has taken several initiatives to resolve the Employee Grievances in shortest period of time. The normal types of Grievances as received by HR along with the durations are shown in Fig. 4. HR delves on establishing an efficient system to address staff enquiries in the area of statutory requirements and regulatory framework. The dynamic nature of the regulatory framework poses a communication challenge to

Table 7: HR newsletter satisfaction rates and preferred sections

\begin{tabular}{lc} 
Category of HR news letter & Satisfaction(\%) \\
\hline MOH and legislative news & 85 \\
Ministry of social affairs news & 96 \\
Bank news & 81 \\
Vacancies & 99 \\
Quotes & 87 \\
New hires photos and description & 93 \\
New services @ the hospital & 89 \\
HR department news & 93 \\
Training updates & 85 \\
Personal accolades & 97 \\
Physicians & 87 \\
Nursing & 83 \\
Technicians & 89 \\
Support services & 92 \\
\hline
\end{tabular}

Table 8: Preference rating by employees with regard to orientation sessions

\begin{tabular}{ll}
\hline Response (\%) & Orientation sessions outcome \\
\hline 55 & They understood the Mission and vision of the organization and department and would align themselves in achieving the same \\
95 & Understood the performance appraisal mechanism including job expectations \\
88 & Understood the legislative and HR operational framework including contractual obligations \\
63 & Understood the disaster management system including the protocols \\
99 & Electrical fire and safety procedures including ergonomics \\
98 & Hospital information system and their concerned working module \\
100 & MS office \\
66 & Professional practice and their role in ensuring patient safety \\
73 & Asset management and purchasing process \\
45 & Orientation to various hazards (chemical, physical, radiological, biological and Bio-Medical) \\
91 & Standards of behaviour would change the way they would communicate with their colleagues and customers \\
75 & Understood their role in contributing towards quality initiatives including accreditation process and role as self assessment team member
\end{tabular}




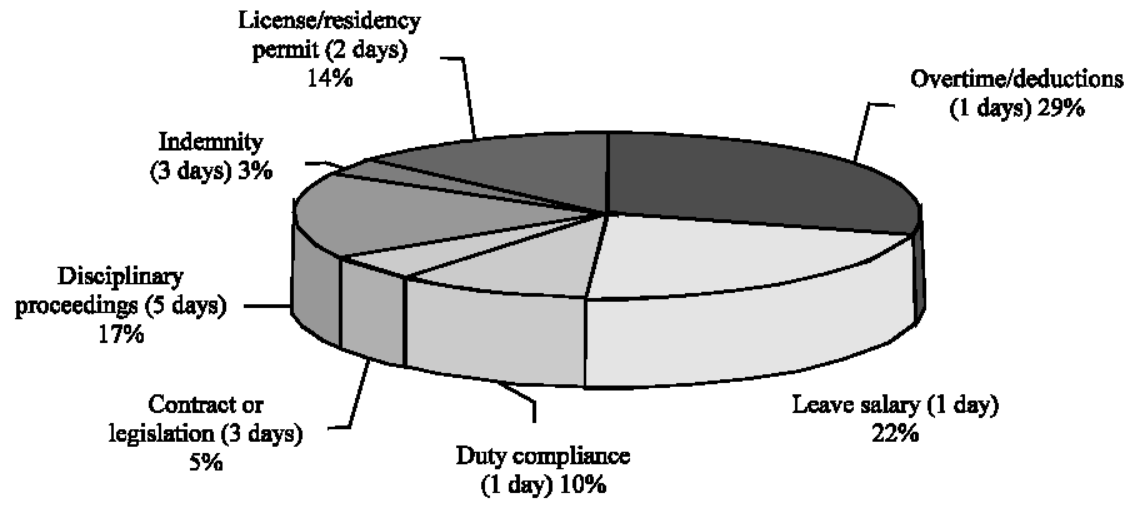

Fig. 4: Grievance resolution (duration) and types

\begin{tabular}{lcll}
\multicolumn{4}{l}{ Table 9: Trainings offered by HR } \\
\hline Category & Sessions & \multicolumn{1}{l}{ Category } & Sessions \\
\hline Stress management & 5 & Communication skills & 13 \\
Decision making & 7 & Creativity in your job & 3 \\
Problem solving & 9 & Project planning and evaluation & 2 \\
Time management & 11 & MS office & 15 \\
Prioritization & 5 & Hospital information sy stem & 17 \\
Leadership skills & 4 & E-mail management & 13 \\
Ergonomics & 5 & Web browsing and content & 7 \\
& & Management & 116 \\
Total & & &
\end{tabular}

Table 10: On-the-job impact of training sessions response from managers Department

Nursing

Reception

Finance

Administration

Marketing

Public relations

Kitchen and cafeteria

Environment and cleaning services

Maintenance

Average HOD response $(\%)$

HR. This challenge is mitigated through seamless communication to employee through circulars, intranet, presentations, informal discussions, HR Newsletter and HR help desk. The result has been tremendous and staff awareness has been raised by $70 \%$ and staff participation has increased by $30 \%$ in these sessions.

Theres an open door policy in place and staff grievances are handled in a sensitive and time efficient manner. Counselling is provided as the first step in the labor management relations. In the last year, there had been 21 instances of employee counselling and as a result of which staff awareness and performance had increased with fewer number of complaints. Above all there are no litigations in courts. There has been remarkable progress in the efficiency, Achievements and Automation of processes in the HR Department. It has again surpassed all the benchmarks set in the HR Balance Scorecard.

\section{CONCLUSION}

Improvement in the magnitude of indicators is observed as a result of the accreditation process.

- Improved Compensation and Benefits for all categories of staff that are regularly bench marked with hospitals of similar stature

- Considerable reduction in Recruitment Cost and Lag including improved Manpower Planning process is observed

- Improved Retention of Employees through enhanced Staff Satisfaction thereby reducing the Impact and Cost of Turnover

- Developed Health and Safety Initiatives by deploying Equipment and Job Hazard Analysis and thereby reducing the injuries

- Improved HR Department Efficiency through Automation of various processes and developed awareness of Legislation, Employer Obligations and Employee Responsibilities

- Ensured ongoing staff competence through ongoing orientation, education and training

A thorough understanding of the accreditation process is achieved and the impact of accreditation on quality of HR processes has been brought into focus.

\section{REFERENCES}

Antonacopoulou, E.P. and L.F. Gerald, 1996. Reframing competency in management development. Hum. Resour. Manage. J., 6: 27-48.

Buchan, J., 2004. What difference does good HRM make?. Hum. Resour. Health, 2: 6-6.

Canada, A., 2006. CCHSA accreditation program, a client centered approach. Human Resources Development and Management Standards, 2006. 
Gifford, B.D., R.F. Zammuto, E.A. Goodman and K.S. Hill, 2002. The relationship between hospital unit culture and nurses quality of work life. J. Healthc. Manage., 47: 13-26.

Holbeche, L., 2001. Aligning Human Resources and Business Strategy. Elsevier Publishing Co., London, New York.

Schuler, R.S. and S.E. Jackson, 2006. Strategic Human Resource Management. Blackwell Publishers, Oxford, England.
Sitawati, R., S. Manaf and E. Winarti, 2009. The Application of Human Resource Scorecard: A Case Study of Public Hospitals in NTT Province Indonesia. IBBA, EuroJournals Inc., Morrisville, North Carolina, pp: 51-66.

Tsasis, P. and B. Harber, 2008. Using the balanced scorecard to mobilize human resources in organizational transformation. Health Serv. Manage. Resour., 21: 71-80. 